\title{
Top rank
}

In 1929, John Slater published a paper entitled "The Theory of Complex Spectra”, in which he applied the emerging mathematics of quantum theory to the physics of complex multi-electron atoms. Among papers published in the Physical Review family of journals over the past century, Slater's paper now ranks $1,853 \mathrm{rd}$ in terms of citations - somewhat lower than might be expected, perhaps, for a landmark in the development of quantum mechanics. All physicists know Slater's name because of this paper, which introduced the 'Slater determinant' for many-body wavefunctions. Why doesn't the citation count reflect this?

We may now have an answer for Slater's paper as well as for other 'classic' papers that the citation count apparently undervalues. It seems there is a better way to use citations in judging the importance of a paper. The key idea, fittingly enough, has emerged out of the technology for searching the World Wide Web.

Google is the world's favourite search engine, largely because of the algorithm it uses to rank web sites by relevance and quality. If you search on keywords, Google ranks sites, in part, according to whether they contain those words, and how they appear on the page. But a page also scores more highly if lots of other pages have links to it, effectively 'voting' for it as being useful. Votes from more highly ranked sites count more. Google scores sites highly if they receive lots of votes from other highly ranked sites.

This circular definition expresses the self-consistency that important pages catch the interest not only of other pages, but of other important pages. Calculating this measure - its 'Google number' - turns out to be equivalent to finding an eigenvector of a matrix reflecting the links between pages.

Clearly this idea might be applied to any network, including that of research papers linked by citations, which is what $\mathrm{Pu}$ Chen and colleagues have now done for papers in Physical Review. The Google idea implies that important papers should not only garner lots of citations, but citations from other important papers. To calculate Google numbers, Chen and colleagues used a database of Physical Review citations for 353,268 articles published from

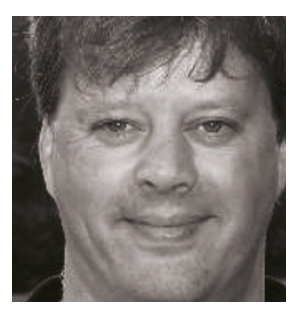

CITATION COUNT IS A BLUNT INSTRUMENT, AS WE MAY HAVE LONG SUSPECTED.

\section{Truth in advertising}

It used to be said that cosmologists were frequently wrong, but never in doubt. That's supposed to have changed now cosmology has become a precision science. Certainly the remarkable data on the cosmic microwave background (CMB) radiation have provided us with a powerful way to explore and constrain fundamental parameters in physics and cosmology.

Many years ago, a colleague argued cogently to me for a theorem of observational cosmology - that it would always be impossible to measure fundamental parameters, such as the Hubble constant, because astrophysical systematic uncertainties would always creep in to overwhelm any clear determination. Thankfully, that has changed. Many new observables, including measures of the largescale clustering of matter, combine to give a stable, often precise, determination of such quantities as the flatness of the Universe and the density of baryons.

But we should not get carried away. For example, one hears that the third year of data from the Wilkinson Microwave Anisotropy Probe (WMAP), provides "stunning" support for inflationary cosmology. Journalists may need to elicit hyperbole from scientists to sell magazines, but the new power spectrum constraints from the $\mathrm{CMB}$ alone are similar to the old constraints from combining $\mathrm{CMB}$ data with other data involving large-scale structure.

We must also remember that some results are wrong. The
1893 to 2003 . Their results reveal some fascinating discrepancies between crude citation rankings and the more sophisticated Google measures (http://arXiv.org/abs/ physics/0604130).

For example, the famous 1944 paper of Lars Onsager, in which he reported the solution of the twodimensional Ising model, ranks only 55th in terms of citations, but rises to 6 th in the Google ranking. This change reflects the fact that papers referring to Onsager's work turned out to be exceptionally important themselves. What about Slater's paper? Ranked 1,853rd purely in terms of citations, it jumps to number 10 in the Google ranking, for similar reasons. The Slater determinant slipped into common usage and into a number of other papers that went on to become classics. Today, this paper gets few direct references, but scores points indirectly in Google terms as others papers that cited it long ago continue to accrue new citations.

So the citation count really is a blunt instrument, as we may have long suspected. There's a better way. One can hope that those who assess scientific output will begin to use it.

Mark Buchanan

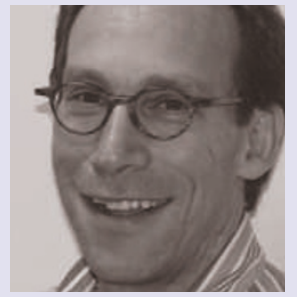

IT USED TO

BE SAID THAT COSMOLOGISTS WERE FREQUENTLY WRONG, BUT NEVER IN DOUBT. significant shift between the first and third year WMAP data in the inferred epoch of re-ionization, when the first stars presumably formed, is one such example.

Current observations are far from finalizing our cosmological paradigms - there is still no unambiguous test of inflation, for example. Meanwhile, the detection of non-zero neutrino masses unleashed a new set of possibilities that might change our understanding of phenomena in the early Universe.

In short, it is possible to celebrate the richness and power of our new windows on the Universe without acting so much like, well, cosmologists!

Lawrence M. Krauss 